Title: A new herpes zoster subunit vaccine for older adults

Charlotte Warren-Gash ${ }^{1 *}$, Judith Breuer ${ }^{2}$

Affiliations:

1. Faculty of Epidemiology and Population Health, London School of Hygiene \& Tropical Medicine, Keppel Street, London, WC1E 7HT, United Kingdom

2. Division of Infection \& Immunity, University College London, 90 Gower Street, London WC1E 6BT, United Kingdom

Correspondence to:

\title{
J.B. j.breuer@ucl.ac.uk
}

A randomized placebo-controlled phase 3 trial of adjuvanted herpes zoster subunit vaccine has shown a substantial improvement in vaccine efficacy in adults aged 70 years and over compared to the current live attenuated vaccine. The finding has profound implications for reducing illness burden, although the duration of vaccine protection needs further evaluation.

Refers to: Cunningham AL et al. NEJM 2016; 375:1019-1032

Herpes zoster causes major morbidity among older people. The incidence of the disease rises dramatically with age, with a lifetime zoster risk for those aged $\geq 85$ years approaching $50 \%{ }^{1}$. Moreover, the risk of post-herpetic neuralgia - a debilitating neuropathic pain syndrome increases markedly after the age of 50 years ${ }^{2}$. Case fatality of herpes zoster is also highly agecorrelated: more than $95 \%$ of deaths occur in people aged $\geq 60$ years ${ }^{3}$. T-cell mediated immunity, which controls reactivation of the varicella zoster virus (VZV) from latency and promotes recovery after herpes zoster, declines progressively with age $\mathrm{e}^{4}$. In addition, cell-mediated immune response to the currently licensed live attenuated zoster vaccine is lower in older age groups, and the effects of 
the vaccine wane over time ${ }^{4}$. Identification of more-effective strategies to prevent zoster among older adults is, therefore, essential.

In a GlaxoSmithKline-funded study, published recently in the New England Journal of Medicine, Anthony Cunningham and colleagues reported impressive efficacy of an adjuvanted herpes zoster subunit vaccine in adults aged 70 years and over 5 .

In the study, known as ZOE-70, 13,900 participants with a mean age of 75.6 years were recruited from 18 countries and randomly allocated to receive either two doses of an investigational herpes zoster subunit vaccine ( $\mathrm{HZ} / \mathrm{su}$; GSK vaccines) or placebo ${ }^{5}$. The new vaccine contained $50 \mu \mathrm{g}$ of recombinant VZV glycoprotein E and the liposome-based AS01b adjuvant system, which enhances CD4+ T-cell and humoral immune responses to recombinant proteins. Over 3.7 years of follow up, only 23 confirmed cases of herpes zoster were observed in the vaccinated group, whereas 223 cases were confirmed in the placebo group, meaning that the vaccine efficacy (VE) for preventing herpes zoster in individuals who had received both vaccine doses was $89.8 \%$ (95\% Cl 84.2- 93.7).

To increase statistical power of the study, authors conducted several prespecified pooled analyses between participants from ZOE-70 and the ZOE-50 trial. The ZOE-50 trial was conducted by the same group of investigators, and used methods identical to ZOE-70 to investigate efficacy of $\mathrm{HZ} /$ su vaccine in adults aged $\geq 50$ years ${ }^{6}$. The results of the ZOE-50 trial were published last year and showed a very marked reduction in herpes zoster among vaccinated participants in this age-group (VE 97.2\% (95\% C.I. 93.7-99.0)). Combined analysis of all $16,596 \mathrm{ZOE}-50$ and ZOE-70 trial participants aged $\geq 70$ years showed a 91.3\% (95\% Cl 86.8-94.5\%) VE against herpes zoster. In another prespecified pooled analysis of ZOE-50 and ZOE-70 trial participants aged $\geq 50$ years, VE against post-herpetic neuralgia was $88.8 \%(95 \% \mathrm{Cl} 68.7-97.1 \%)$. 
The choice of primary end point in the present study - reduction in herpes zoster incidence - is supported by a recent review of endpoints in zoster vaccine studies ${ }^{7}$, which concluded that use of the main alternative endpoint, burden of illness (determined by averaging pain severity and duration scores based on the Zoster Brief Pain Inventory), tends to overestimate VE ${ }^{7}$. Although other studies have considered the effect of zoster vaccine on activities of daily living or health-related quality of life, the main effect of zoster vaccine on these endpoints is through preventing zoster, rather than by attenuating severity or duration of illness ${ }^{7}$, which supports the approach Cunningham and coinvestigators took.

The results of ZOE-70 are noteworthy for several reasons: first, they demonstrate a marked increase in efficacy over the current vaccine, which is licensed for adults aged 50 years and over. This is a live attenuated Oka/Merck VZV vaccine (Zostavax), shown in the landmark Shingles Prevention Study to reduce herpes zoster incidence by $51.1 \%$, burden of illness attributed to shingles by $61.1 \%$, and post herpetic neuralgia by $66.5 \%^{8}$. Second, efficacy of the new subunit vaccine did not diminish with advancing age: VE was $90.0 \%$ in those aged $70-79$ years and $89.1 \%$ in participants aged 80 years or older. This compares favourably to age-stratified VE estimates for the current live attenuated vaccine of $69.8 \%$ in participants aged 50 to 59 years ${ }^{9}, 63.9 \%$ among subjects aged $60-69$ years and $37.6 \%$ among those aged 70 years and over $^{8}$ for reducing herpes zoster incidence.

Establishing an adequate immune response to vaccines in older people is challenging: in general, the size, duration and quality of response, regardless of vaccine type, is lower than in younger people ${ }^{4}$. The high efficacy of the new $\mathrm{HZ} / \mathrm{su}$ vaccine suggests that the vaccine is able to overcome some of the processes associated with senescence of adaptive immunity, notably the high background inflammatory state implicated in age-related alterations to T-cell immunity ${ }^{10}$, which can inhibit T-cell activation following vaccination in older people. Better understanding of the biology underlying the 
findings from ZOE-70 could, therefore, have more general implications for improving vaccine efficacy in the elderly

Besides older people, the $\mathrm{HZ} / \mathrm{su}$ vaccine could benefit other groups with impaired cell-mediated immunity - such as those individuals on immunosuppressive therapies or with various haematological malignancies - who may be at high risk of herpes zoster but in whom live vaccine is contraindicated. $\mathrm{HZ} /$ su vaccine is not a live vaccine and as such, the virus cannot replicate. The vaccine can, therefore, theoretically be used in immunocompromised people, although this was not tested in the trial, and safety and efficacy in this group would require further investigation.

One potential disadvantage of the $\mathrm{HZ} / \mathrm{su}$ vaccine is the higher frequency of solicited injection-site and systemic reactions, most frequently fatigue, reported within 7 days of the vaccine compared with placebo (79.0\% versus $29.5 \%)$. These adverse reactions tended to be transient, mild to moderate reactions, with a median duration of 2-3 days for injection site reactions and 1-2 days for systemic reactions. Systemic reactions were more frequently reported in $\mathrm{HZ} /$ su vaccine recipients $(53.0 \%)^{5}$ than in recipients of live attenuated vaccine $(25 \%)^{8}$. Importantly, rates of serious adverse events, immune-mediated disease and deaths did not differ between $\mathrm{HZ} /$ su vaccine and placebo groups. Another potential issue is compliance: the subunit vaccine requires two doses given 2 months apart, rather than one dose as is the case for live attenuated vaccine. Encouragingly, VE results for the total vaccinated cohort (including people who received only one vaccine) remained high, with VE of $87.7 \%$ (95\% Cl 82.0-92.0\%).

The duration of protection provided by the $\mathrm{HZ} /$ su vaccine remains an open question. Efficacy of the live attenuated vaccine declines markedly over the post-vaccination years 3 to 11 . Although waning of $\mathrm{HZ} /$ su vaccine-induced immunity will start from a higher baseline, accurate assessment of the likely duration of protection is of key importance to inform cost-effectiveness estimates and, 
ultimately, the design of effective public health programmes. The $\mathrm{HZ} /$ su vaccine offers the exciting prospect of seriously reducing the population burden of zoster complications, especially in settings that already achieve a relatively high vaccine uptake.

1. Hope-Simpson RE. The nature of herpes zoster: a long-term study and a new hypothesis. Proc. R. Soc. Med 1965; 58: 9-20.

2. Forbes $\mathrm{HJ}$, et al. Quantification of risk factors for postherpetic neuralgia in herpes zoster patients: A cohort study. Neurology 2016; 87(1):94-102.

3. Hobbelen PHF, Stowe J, Amirthalingam G, Miller L, van Hoek A-J. The burden of hospitalisation for varicella and herpes zoster in England from 2004 to 2013. J Infection 2016; 73:241-253.

4. Levin M. Immune senescence and vaccines to prevent herpes zoster in older persons. Curr Opin Immunol 2012; 24: 494-500.

5. Cunningham AL, et al. Efficacy of herpes zoster subunit vaccine in adults 70 years of age or older. NEJM 2016; 375(11):1019-1032.

6. Lal $\mathrm{H}$, et al. Efficacy of an adjuvanted herpes zoster subunit vaccine in older adults. NEJM $2015 ; 372(22): 2087-96$.

7. Cook SJ, Flaherty DK. Review of the persistence of herpes zoster vaccine efficacy in clinical trials. Clinical Therapeutics 2015; 37(11): 2388-2397.

8. Oxman MN, et al. A vaccine to prevent herpes zoster and postherpetic neuralgia in older adults. NEJM 2005; 352(22): 2271-2284.

9. Schmader KE, et al. Efficacy, safety and tolerability of herpes zoster vaccine in persons aged 50-59 years. Clin Infect Dis 2012; 54(7): 922-8.

10. Macaulay R, Akbar AN, Henson SM. The role of the T cell in age-related inflammation. Age (Dordr) 2013; 35: 563-572. 
Competing interests statement

No competing interests declared. 